\title{
COAXIAL DISC WINDOWS FOR A HIGH POWER SUPERCONDUCTING CAVITY INPUT COUPLER
}

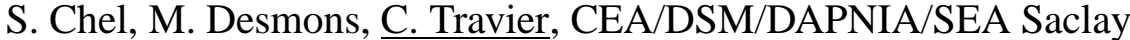 \\ T. Garvey, P. Lepercq, R. Panvier, CNRS/IN2P3/LAL Orsay
}

\section{Abstract}

A $\lambda / 2$ coaxial L-band window has been tested up to $1 \mathrm{MW}$ ( $1 \mathrm{~ms}$ pulses), both at room temperature and at $80 \mathrm{~K}$. Dielectric losses were measured. A travelling wave window with lower dielectric losses has been fabricated.

\section{INTRODUCTION}

In the framework of the TESLA collaboration, Saclay and Orsay are designing an alternate input power coupler. The aim is to develop a simplified version of the present TTF coupler, in order to reduce the cost. The way to achieve this simplification is to consider a fixed (non tunable) and rigid (non flexible) coupler. As part of this effort, a coupler test stand including a $80 \mathrm{~K}$ cryostat was constructed [1], and several coupler parts were designed and tested. This paper presents the experimental results concerning a $\lambda / 2$ disc window. It also describes the design of a travelling wave (TW) disc window.

\section{$2 \lambda / 2$ DISC COAXIAL WINDOW}

The simplest coaxial window one can imagine is a ceramic disc brazed inside the $50 \Omega$ coaxial pipe. In order to be selfmatched, the ceramic should be half a wavelength thick. Using WESGO Al300 alumina which has a permittivity $\epsilon_{r}=9$ at the designed frequency of $1.3 \mathrm{GHz}$ (as given by the vendor), the window was designed with the parameters given on table 1. The inner conductor is made of copper, while the outer conductor is made of kovar, an alloy which has a linear thermal expansion coefficient very close to that of the ceramic and is therefore often used in ceramic/metal assemblies. The kovar piece is then TIG welded to the stainless steel copper coated outer conductor as shown in fig. 1. The ceramic is coated with a thin layer of TiN. This window was fabricated by SICN [2].

The main advantages of this type of window are its relative simplicity, its robustness, the absence of electric field perpendicular to the ceramic surface which is favorable for avoiding multipactor, and the ease with which the window can be cleaned prior to its assembly on the cavity. The potential drawbacks are the high electric field at the braze location and the high dielectric losses due to the large volume of ceramic used.

\section{WINDOW TEST CONDITIONS}

The window was mounted on the test stand presented in reference [1]. It is inserted on the test line between a door-
Table 1: Windows characteristics

\begin{tabular}{|c|c|c|}
\hline \multicolumn{3}{|l|}{ Characteristics } \\
\hline & $\overline{\lambda / 2}$ & TW \\
\hline Inner diameter $(\mathrm{mm})$ & 26.8 & 75.6 \\
\hline Outer diameter (mm) & 61.6 & 142.2 \\
\hline Ceramic thickness (mm) & 38.4 & 10 \\
\hline \multicolumn{3}{|l|}{ Performances } \\
\hline Bandwidth $(\mathrm{MHz})(\mathrm{S} 11<0.1)$ & 80 & 80 \\
\hline Ratio between max field at bra- & & \\
\hline zing and max field in coax & 1 & 0.2 \\
\hline Avg. dielectric losses (W) & & \\
\hline at $1 \mathrm{MW}$ (TESLA pulse) & 44 & 6.5 \\
\hline
\end{tabular}

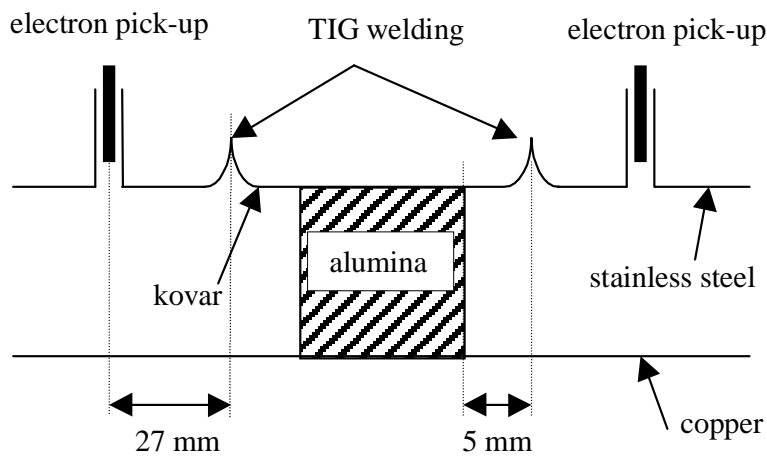

Figure 1: $\lambda / 2$ disc coaxial window

knob type and an antenna type waveguide to coax transition as shown schematically on fig. 2 . The diagnostics used are 2 electrons pick-ups located on both sides of the window and 2 photomultipliers located as shown on fig. 2 .

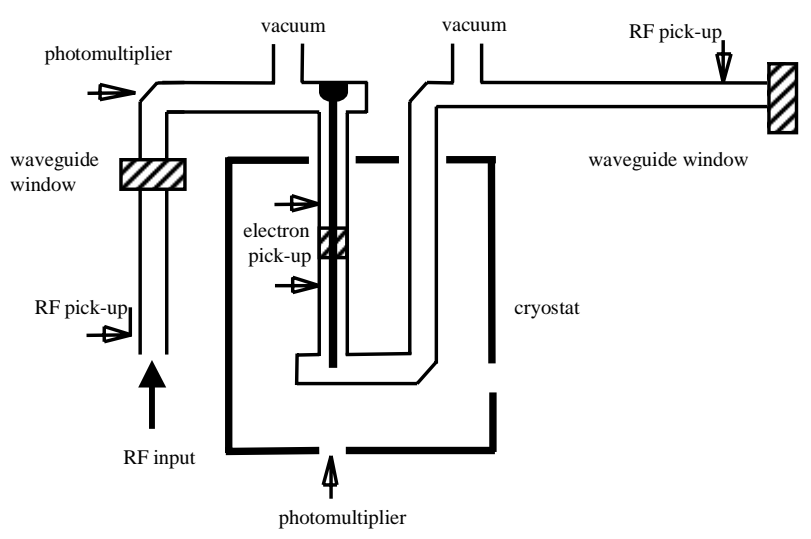

Figure 2: Coupler test stand 
The present capability of the power source allows tests at a peak power of $1 \mathrm{MW}$, for pulses of $800 \mu \mathrm{s}$ and a repetition rate of $0.1 \mathrm{~Hz}$. The system runs continuously and all signals (RF power, vacuum, electrons, light, temperatures) are recorded for each pulse. This $\lambda / 2$ window was tested for almost 5 months between August 1998 and January 1999 , this period corresponding to 990,983 pulses. For each pulse, 80 parameters are recorded, so that the total amount of data represents 1.3 Gbytes. Since the analysis of such a huge amount of data cannot be made with standard spreadsheet softwares, PAW [3] is used.

The window was tested under all possible conditions: at room temperature and at liquid nitrogen temperature, with standing and travelling wave rf input, with vacuum on both side and with atmospheric pressure on one side. The result of all these tests are presented in the next sections.

\section{TEST UNDER STANDING WAVE CONDITIONS}

By using a short-circuit at the end of the line, one can operate the test stand under standing wave conditions thus simulating what is happening during the filling of the cavity. The initial conditioning of the window was done under these standing wave conditions. It took nearly 30,000 pulses to reach the $1 \mathrm{MW}$ level. At that point, while the system was processing at $1 \mathrm{MW}$, an incident occurred that exhibited large electron, light and vacuum signals, downstream of the window. Unfortunately, the software interlock that would normally reduce the input power in such case didn't work. As this event happened at the beginning of the week-end, the system ran for the whole week-end at $1 \mathrm{MW}$. Due to large electron emission at or near the downstream side of the window, the power was fully reflected at this location. After that unfortunate event, it took more than 300,000 pulses to recondition the window up to $1 \mathrm{MW}$. Once conditioned, we cannot observe any systematic and significant electron or light signal at any power level. At a very erratic rate, one can see some electron signal when the electric field is maximum at the window surface, especially on the side that was potentially damaged by the event described above. To illustrate this behaviour, fig. 3 shows that, while running at $1 \mathrm{MW}$, some strong electron signal can be seen. After a few tens of pulses these signals are processed away. The figure shows that these multipacting events are seen on the vacuum recording, on the light signal and on the temperature indicator located on the outer conductor in the vicinity of the window. However, all these signals do not allow one to know precisely if the emission is occurring exactly at the window surface (braze location) or somewhat further in the conductor and especially at the kovar/stainless steel weld that is only $5 \mathrm{~mm}$ downstream. The behaviour of the window under standing wave conditions is exactly the same both at room temperature and at $80 \mathrm{~K}$.

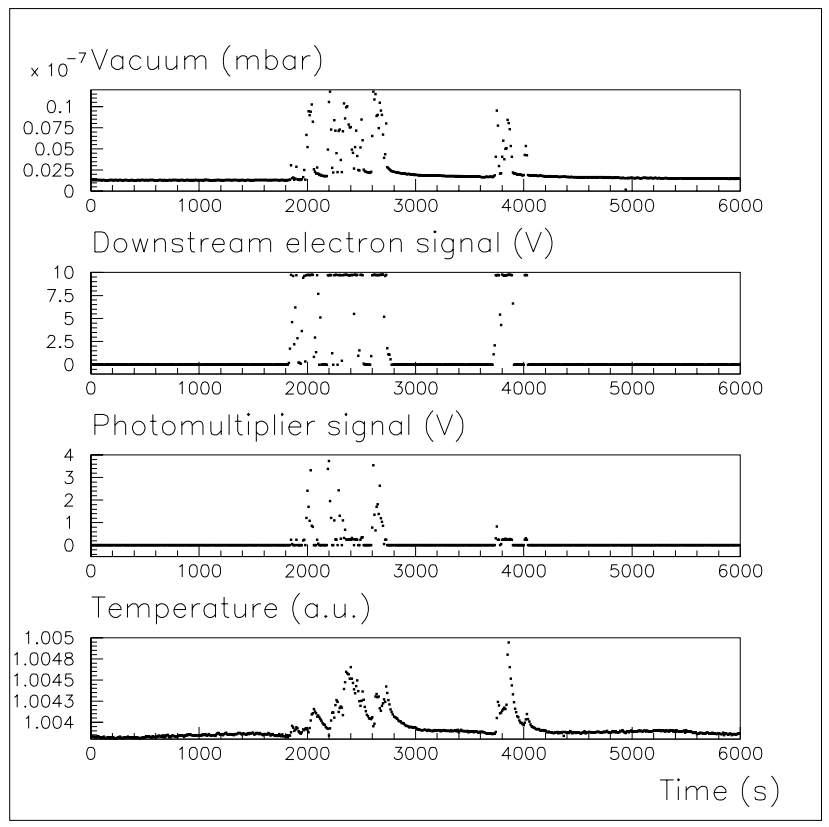

Figure 3: An example of multipactor behaviour at $1 \mathrm{MW}$ (standing wave conditions)

\section{TEST UNDER TRAVELLING WAVE CONDITIONS}

Under travelling wave operation, one can observe electron activity on both sides of the window. However as shown on fig. 4, the signal is much stronger and the power range is wider on the damaged side. Fig. 4 also shows that this multipactor can be processed: after 180,000 pulses, signal was strongly diminished on the downstream damaged side and completely disappeared on the upstream side. We didn't wait long enough to see, if it would also disappear on the downstream side. Here again, the behaviour is similar at room temperature and at $80 \mathrm{~K}$.

\section{DIELECTRIC LOSSES IN THE WINDOW}

Since the window is thermally connected to the liquid nitrogen cooled shield, the total heat load it produces is of major concern. In the case of the $\lambda / 2$ window, the main part of the heat load is due to the dielectric losses. By choosing properly the distance between the superconducting cavity and the window itself, the dissipation at LN2 temperature can be reduced. For the complete TESLA pulse (filling time of $530 \mu \mathrm{s}$ and beam time of $800 \mu \mathrm{s}$ ), the dissipated power Pdiss in the dielectric is evaluated to be: $8.79 * \tan \delta * \mathrm{Av}-$ erage incident power with a distance $=(2 \mathrm{~N}+1) \lambda / 4$ and 5.83 $* \tan \delta *$ Average incident power with a distance $=\mathrm{N} \lambda / 2$. In the standing wave case and for a distance corresponding to $(2 \mathrm{~N}+1) \lambda / 4$, we measured a dissipation corresponding to $1.1 \%$ of the average input power, from which we infer that $\tan \delta=5.810^{-4}$. 


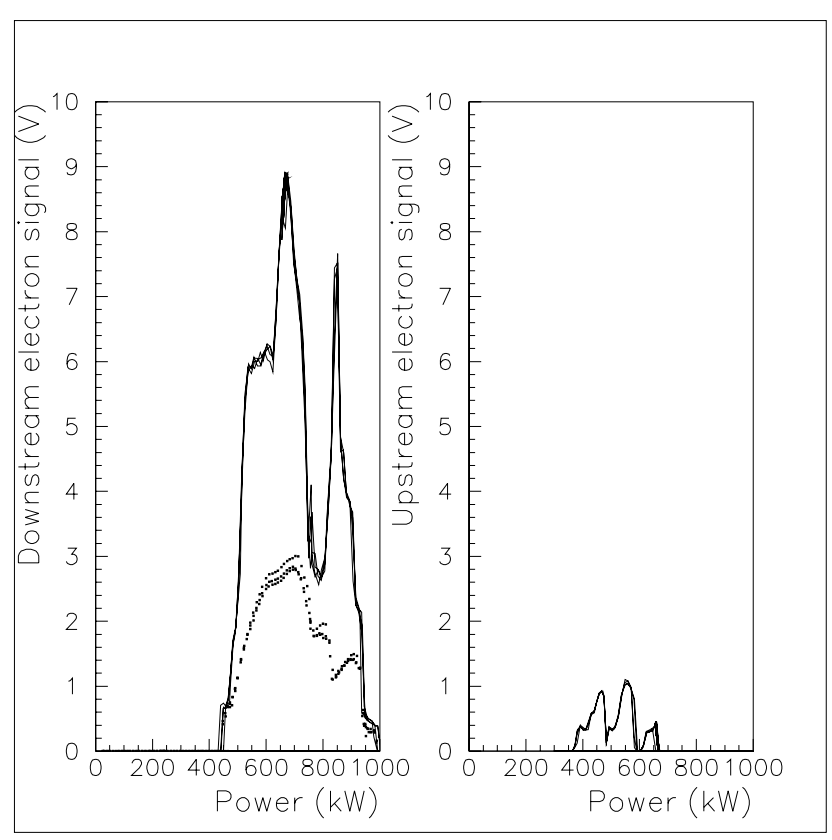

Figure 4: Multipactor as a function of power for TW operation, upstream and downstream sides: at some time (line) and 180,000 pulses later (dots).

\section{TRAVELLING WAVE WINDOW}

In order to reduce the dielectric losses in the ceramic, one can use a thinner ceramic and/or reduce the electric field inside the ceramic. With a thin ceramic, the matching is achieved by adding some inductive or capacitive components on the coax on both side of the ceramic. The simplest solution is to use some bumps on the coaxial inner conductor as proposed in reference [4]. Here, we are trying to find a design where the ceramic would be completely shielded from the electrons coming from the cavity to avoid the potential problems seen, for example, at CEBAF [5]. The advantages of the window, shown on fig. 5, are a low field inside ceramic and therefore low losses, a low field at the brazing location, no field components perpendicular to the ceramic surface, no direct exposure of the ceramic to cavity electrons, while the main drawback are its relative complexity, its large diameter, and the difficulty to clean it. Two windows that are now ready to be tested, were fabricated by SICN [2]: one where titanium is used for the outer conductor brazed on the ceramic and the other for which copper is used. In both cases, the inner conductor is made of plain copper.

\section{CONCLUSION}

In this paper we present the results of the high power tests made on the coaxial $\lambda / 2$ window that we designed and fabricated. The first results are encouraging. Though a failure of the control system at the beginning of the conditioning led to a metallic coating of one side of the window (the precise nature of this coating is under investigation), it was later on capable of sustaining $1 \mathrm{MW}$ of peak power, both

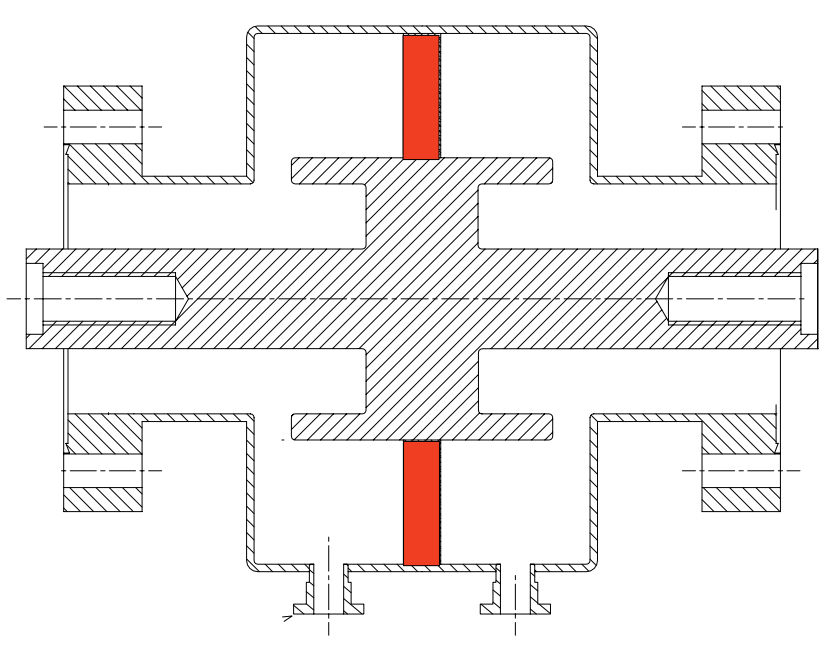

Figure 5: Travelling wave window

under travelling and standing wave operation, both at room temperature and at $80 \mathrm{~K}$, and both under vacuum and atmospheric pressure. Up to $400 \mathrm{~kW}$, the window works without any signs of electron activity. Between $500 \mathrm{~kW}$ and $1 \mathrm{MW}$, the travelling wave operation reveals some electron activity that we cannot attribute with certainty to the window. It will be necessary to test a second, identical, window that was fabricated to confirm these results. It will then be necessary to fabricate a new window with a slightly different design (replace kovar by titanium or copper, and replace the TIG welding by electron beam welding) to see whether it is possible to eliminate electron activity over the full power range.

The dielectric losses were measured and proved to be too high for operation at the TESLA repetition rate and peak power needed for the superstructures [6]. To improve this situation, it would be necessary to use ceramics with a higher purity. These ceramics exist but the brazing to metal is more difficult. Another solution is to use the travelling wave window that was presented in this paper, and which will soon be tested.

\section{ACKNOWLEDGEMENTS}

We would like to thank all the people at IN2P3 and DAPNIA involved in this work for the design, construction and operation of the test stand, especially F. Blot, D. Braud, P. Dufresne, A. Hamdi, M. Kalmykow.

\section{REFERENCES}

[1] S. Chel et al., EPAC98, p. 1882.

[2] SICN, BP 1, 38113, Veurey-Voroize, France.

[3] PAW, CERN Program Library Long Writeup Q121, 1995.

[4] X. Hanus, A. Mosnier, Proc. of the $7^{\text {th }}$ Workshop on RF superconductivity, Gif sur Yvette, October 17-20, 1995, p. 701.

[5] T. Powers, P. Kneisel, ibid, p.. 713.

[6] M. Ferrario, J. Sekutowicz, EPAC98, p. 1876. 